\title{
The Pedagogy of Visual Discourse: An Analytical Approach to Teaching and Evaluating the Rhetorical Image
}

\author{
Jacqueline Irwin \\ California State University, Sacramento, USA \\ Email: jaccie.irwin@gmail.com
}

Received 15 February 2014; revised 17 March 2014; accepted 25 April 2014

Copyright (C) 2014 by author and Scientific Research Publishing Inc.

This work is licensed under the Creative Commons Attribution International License (CC BY). http://creativecommons.org/licenses/by/4.0/

(c) (i) Open Access

\begin{abstract}
As the area of visual rhetoric develops and evolves, the approaches that critics take in evaluating images must be scrutinized for the overall exploration of the discipline. Incorporating areas of analytical criticism from rhetoric to aesthetics to design should be combined to create the best possible way of evaluating imagery. By expanding on the traditional analytical approach to rhetorical criticism, this paper explores how the additional understanding of aesthetic and design theory will help the critic to reach a fuller understanding of the image. The twelve major principles of design being line, shape and form, space, texture, value, color, repetition, variety, rhythm, balance, emphasis, and economy are combined to create the strategy of the visual aesthetic that works to compliment the existing rhetorical strategies. The more complete understanding of how visuals are created and how people interpret them will allow for a more complete development of the visual rhetorical approach to communication.
\end{abstract}

\section{Keywords}

Visual Rhetoric, Methodology, Aesthetic and Design Theory

\section{Introduction}

As the study of rhetoric enters the twenty-first century, the definition of what constitutes a rhetorical text has changed. The Internet and other visual media have invaded our society and are demanding attention from rhetorical scholars. The twenty-first century has developed a new consumer that is bombarded with thousands upon thousands of visual images on a daily basis. Consumers as well as critics are in need of an approach by which to critically evaluate the persuasive strategies used in these messages. Organizations need to be aware of the visual 
rhetorical strategies available to them in order to reach their specific audiences. Employing principles found in design theory as rhetorical strategies, organizations can design more effective messages to reach their desired audiences. In this paper, the current study of visual rhetoric will be explored; an outline of the visual rhetorical strategies and explanations for each will be developed; and then an analysis of an online organization's website will be evaluated in regard to the established strategies. Utilizing this method of strategic visual message design will lead not only to the creation of better visuals, but also to a better understanding of the visuals that persuade us everyday.

\section{Visual Texts}

Rhetorical analysis had evolved so that no longer is discourse limited to the medium of the spoken word. Brummett states that "the conception of rhetoric as [a purely] verbal text... in which public controversy primarily took oral or written form... is fading" (Brummett, 1991: p. ix). Rhetoric can and is being created and "recorded in the dialects of marble, steel, clay, glass, paper, and ink, not to mention oils, pastels, and acrylics" (Brown, 1983: p. 11). Although rhetorical critics may feel nostalgic for the days when spoken public discourse had the most impact, they are beginning to take notice that "to confine their study of symbols to speech making is to miss a great many of the symbols that affects us daily" (Foss, 1994: p. 213). Studying visual imagery and art from a rhetorical perspective gives scholars a more complete and richer understanding of the rhetorical process because it offers explanation of all of the symbols that surround us, not just the verbal ones.

Visual rhetoric has traditionally suffered from a lack of study because traditional rhetorical scholars have historically believed that visual rhetoric is less important than verbal communication. "Some scholars of rhetoric and contemporary culture believe that imagery as a rhetorical form is tainted [as compared to] discourse in the effects it produces on the nature of public communication and the modes of critical processing it engenders in audiences" (Foss, 1994: p. 222). Many scholars have defined visual artifacts to be of lesser value than verbal communication because they claim that visual rhetoric shortchanges public argument (Postman, 1985; Zarefsky, 1992; Jamison, 1988, 1992). On the other hand, more recently, rhetorical scholars have employed visuals as data in the rhetorical sense by applying them to illustrate various rhetorical constructs. For example, scholars have used Central Park (Rosenfield, 1989); Disneyland (Foss \& Gill, 1987); Michael Graves’ architecture (Kanengieter, 1991); the Vietnam Memorial (Haines, 1986; Foss, 1986); photography (Finnegan, 2003); and interior design (Twigg, 1992) as the basis for applying the traditional rhetorical constructs to visual artifacts.

Scholars in communication studies and in other fields have examined the visual through the application of the gestalt principles (the psychology that the parts of a visual are constructed by the audience into a whole object) and applied them to the organization of visual materials (Bernhardt, 1996; Moore \& Fitz, 1993). This approach seems to be practical because the principles are viewed as axiomatic by researchers to be universal in nature. The gestalt principles are tools that are undoubtedly understood by the rhetor or designer, but are in general not consciously comprehended by the audience (Kostelnick \& Hassett, 2003). Being able to comprehend both the tools and the context of the visuals will lead to a more complete understanding of visual symbols.

One area that has examined the visual is the field of aesthetics. However, many perspectives that are used in aesthetic judging of imagery are not useful in the rhetorical evaluation of images because they overlook the audience and the context of the artifact. For example, one perspective of the evaluation of visual imagery in aesthetic "defines judgments of quality solely as idiosyncratic responses-judgments about quality mean simply preference, rooted in individual whim or fancy" (e.g. Bayley, qtd. in Fuller, 1984). Another perspective is based on the concept that there can be a set of unique criteria for aesthetics (Foss, 1994), but these types of approaches do not particularly help us understand the potential persuasiveness of a particular artifact or how they function rhetorically.

Content, audience, and context function secondarily to the aesthetic quality when rhetorically evaluating the aesthetic experience hence limiting the potential scope of the aesthetic evaluation. "Appreciating something aesthetically [is] to appreciate its formal qualities, those qualities that one could access simply through looking, hearing, [or] touching" (Fenner, 2003: p. 41). When viewing anything from a purely aesthetic perspective, whether it be a work of fine art or a television commercial, the aesthetic critic must attempt to disregard the content of the work and focus on what strategies are used to convey the message. This is why the purely aesthetic approach is lacking in the evaluation of the visual rhetorical message. The rhetorical critic's evaluations may seem irrelevant when that evaluation is viewed as a personal preference. The aesthetic approaches to evaluation are incomplete approaches for rhetorical analysis because while they attempt to identify just the artistic or aes- 
thetic qualities; they are not concerned, as the rhetorical critic is, with the influence of the image on an audience and the ways in which the image is constructed to achieve such an influence. The combination of these approaches will give critics in both fields a more complete understanding of the artifacts they are evaluating.

In order to bring together the visual and its context, one must bring together the tools of different disciplines. The generally misunderstood term visual rhetoric is a compilation of a broad range of areas of study including everything from communication to design to psychology. For example, these can include, "the study of the design of texts on pages" to "more generally, the study of all visual signs, including the semiotics of graphical arts, television, and other media" (Bernhardt, 1996: p. 746), to an even more broad study of "visual and material practices, from architecture to cartography, and from interior design to public memorials" (Lucaiates and Hariman, 2001: p. 37). No matter how different each definition of visual rhetoric is, they all bring to the table a distinction between the image and the word, that is, the ever-present dichotomy of the presentational and the discursive (Langer, 1988). The areas of the rhetoric of the word, the discursive, and the rhetoric of the image, the presentational, have been decidedly divided areas, even though combining them would give both a more complete understanding of all rhetoric. "Written language, Plato observed in the Phaudrus, 'rolls around everywhere' (p. 86), but visual language is even more fluid" (Kostelnick \& Hassett, 2003: p. 10). The root of all rhetoric is our symbolic understanding, and grasping that foundational root is the only way to go about fully grasping the importance of the visual symbol.

The creation of visual rhetoric will always begin with the rhetor or designer using strategies of the visual language to meet a specific audience and then finishes with the audience interpreting and understanding that visual language. Another consideration of the process of critiquing visual rhetoric is that each individual visual rhetorical act is set in larger social set of rhetorical events and the critic must consider both the visual artifact and its context. Just as rhetoric is a social process, so is visual rhetoric. It builds and develops cultural values, visual literacy, and reinforces social norms. "Information design also embodies the shared cultural knowledge-values, ideologies, and aesthetic tastes - of its designers and readers at a given historical moment" (Kostelnick, 2004: p. 239). The social factors that surround visual rhetoric are the foundation for it. Therefore, the critic must take into consideration the context of each visual in their rhetorical evaluation.

\section{Visual Rhetorical Strategies}

Robert Rowland in his book Analyzing Rhetoric, states that "in order to understand the way that any work of rhetoric functions, the analyst needs [a set] of tools. The first set of tools is used to break the rhetoric down into constituent parts. A system of analysis is needed that can be applied systematically to any work of rhetoric" (2002, p. 15). Rowland establishes categories of strategies that are used in the evaluation of any piece of rhetoric. These include: rational argument, narrative, language aesthetics, appeals to values, needs, and symbols, credibility, and confrontation. Each strategy contains specific sub-strategies that further define the ways that rhetoric uses each strategy. In order to fully evaluate a visual artifact the analyst needs another, specific strategy category, that of the visual aesthetic. The visual aesthetics strategy category also has sub-strategies, each of which contributes to the whole of the visual aesthetic. The twelve sub-strategies are: line, shape and form, space, texture, value, color, repetition, variety, rhythm, balance, emphasis, and economy. "Most people already know a great deal about the art of design, but few of us recognize our own visual sophistication" (Zelanski \& Fisher, 1996: p. 2). Taking the time to understand what visuals are being presented to us not only will make the critic more apt at discovering the rhetorical strategies embedded in messages, but will also allow the consumer to make better choices in regards to the messages being presented to them.

The twelve sub-categories are standard principles in design with many scholars developing some or all of them in various forms. Introduction to design courses flourish with these principles to train young artists to rethink about the visual they are creating and looking at. Lauer and Pentak (2000) define these design principles in their text Design Basics (2000) and Lawson (1980) and Margolin (1989) use them in explanations of the history of design theory and discourse. Even in their discussion of the semiotics of visual communication, Kress and Van Leeuwen (1996) utilize the ideas of the design principles in their development of the grammar of visual design. Paul Zelanski and Mary Pat Fisher have written several books including Color (1990), Shaping Space (1987), and Design Principles and Problems (1996) and in these works, they have developed the twelve design principles in the most clearly discernable fashion and thus they are utilized for the definitions of these principles. Thus it is their set of principles that I propose to be used for rhetorically analyzing visual artifacts.

"To design is to endow optical sensations-or images—with meaning”, (Zelanski \& Fisher, 1996: p. 2) and 
we comprehend these messages constantly and unconsciously. As humans, we refuse to view the world as a chaotic jumble of meaningless stimuli. Instead we absorb incoming stimuli and assign them clear meanings. We develop and build this visual vocabulary without even realizing it and it becomes as second nature to us as our verbal vocabulary. "Just as we do not usually stop to think of words as we speak, we do not usually puzzle over the meaning of the things we see" (Zelanski \& Fisher, 1996: p. 3). They argue that for artists to communicate both rhetorically and visually, you must understand how to create and manipulate images so that they communicate to the audience the message that you intend. In order to do this, you must bring back into your consciousness the strategies that you now overlook. "For example, if you want people to think that a flat image you are creating had depth, you must be aware of all the visual cues you have learned to interpret automatically as depth. Awareness of these cues gives you ways of controlling others' perceptions-of making them respond to your flat image as though it had depth" (Zelanski \& Fisher, 1996: p. 3). Seeing and understanding what has always been there is the key to understanding the creation and development of visual messages.

The visual rhetorical strategies stem from the fields of art and design. These visual "cues and perceptual responses are expressed as principles, or basic truths, that the artist can consciously use" (Zelanski \& Fisher, 1996: p. 3). Understanding how the artist uses these principles, or strategies, will allow the rhetorical analyst to more clearly decipher the messages that are embedded in the visual cues. The best way to develop an awareness, understanding, and control of the messages within the visuals is to isolate those most basic strategies and understand how they work one at a time. It is impossible to create an image with just one of these strategies, but just as the repetition of a word does not make speech, repetition is used as a tool to develop the speech. Again, most audiences will not be aware of these visual aesthetic strategies, just as most are not aware of the many language aesthetic strategies, but the analyst must be consciously aware in order to be able to both evaluate and create visual rhetoric.

When a piece of visual rhetoric is working well, the artist is exercising control over how the audience both view and respond to the piece. One way that the artist must work to control is how long a person looks at the piece. We know that speakers are likely to hold an audience's attention for a good portion of a speech. "In contrast, people [tend to] respond to the visual arts very quickly and then turn away. [In] advertisements, the designer must hold viewers' attention long enough for a message to register, or people will not buy the product" (Zelanski \& Fisher, 1996: p. 17). The artist can control how long and what the audience sees by utilizing the visual rhetorical strategies. They can use lines, shapes, and even colors to control and direct the view of the audience so the audience can grasp the message that the artist intends.

Each of the strategies build upon one another and are separated into two groups: strategic elements and unifying principles. The strategies are discussed in an order so that one leads into the other in order to build a unified visual. Line comes first because the creation of the line is the simplest way of creating a visual image and the others are added in logical order: "lines connected make flat shapes; shapes put together in space seem to suggest form. When forms are indicated a feeling of space becomes stronger. Texture bridges the gap from the eye to the touch. Value-the degree of darkness or lightness-refines responses to shape, form, space, and texture and provides the understructure for understanding color. Color adds a special seasoning, a richness, to our visual sensations" (Zelanski \& Fisher, 1996: p. 6). The unifying principles are used simply to create a sense of unity and order to create relationships between parts of the whole image. These relationships are created through the use of similarity, variety, rhythm, balance, emphasis, and economy.

\subsection{Line}

Line begins the strategies because it is the most simple and is the one that the rest are created from. The line may be the simplest of the design principles, but it can be extremely powerful. The problem with line is being able to define what is and what is not a line. When does a line stop being a line and become something else? There are many lengthy definitions of what constitutes a line, but "a paraphrase of Paul Klee's definition is, 'A line is a dot out for a walk'. In general, a line is a mark whose length is considerably greater than its width" (Zelanski \& Fisher, 1996: p. 56).

Lines can be created with just about any medium. When we first think of line, we probably think of those made with a pen or pencil or a brush, but sculpture and even fabrics can have strong linear elements. Lines can be implied in the contrasts between light and dark areas without being actually drawn at all. Lines can be incredibly expressive and persuasive as the way that a line is drawn out from a point is what gives it a certain character. Line has the power to control the audience's view of a visual work as it directs the viewer's eye as 
Zelanski and Fisher state, "If a line has a smooth surface, the eye travels along it easily; if rough or broken, it slows the eye. Lines may be tightly controlled with small hand movements or freely gestured through space" (Zelanski \& Fisher, 1996: p. 63). These characteristics have a tendency to create emotional responses in the audience. Lines that rise and fall with sharp points can suggest anger or danger to the audience, while horizontal lines can read as calm.

Lines are the basic element for the development of any piece of visual rhetoric and understanding that an artist can use a simple line to direct the audience's view to a particular area or create a particular mood with an audience helps in understanding the foundation for the visual aesthetic strategy.

\subsection{Shape and Form}

The second strategy is shape and form. Lines are used to create shapes and shapes are grouped together to create forms. In other words, "a shape is a figure that appears to be flat, whereas a form is a figure that appears to be three dimensional" (Zelanski \& Fisher, 1996: p. 86). Artists have a virtually unlimited amount of resources for inspiration of shapes including geometric shapes, invented shapes, letters, and numbers. Shapes that we learn as children, such as squares, circles, triangles, and the like, are the most familiar to the audience and the artist can use that familiarity to their advantage in controlling the view of and creating a mood in the audience. However, because of this familiarity of the geometric shape, audiences can have preconceived notions about what these shapes mean and will do. Circles can look as if they are rolling away unless held in position by something else. Triangles can seem out of balance if their bases aren't perfectly parallel to the bottom of the picture (Zelanski \& Fisher, 1996: p. 88).

Irregular shapes demand more attention than geometric shapes and can be used to control the attention of the viewer. Invented shapes give the artist more freedom than geometric shapes because the audience isn't familiar with them or how they may behave. The logical use of these shapes doesn't seem to be supported by anything else, but this is not uncomfortable for the viewer, as they seem to come from the artist's imagination. The use of invented shapes may give the artist more freedom, but then the artist may not be able to interpret how an audience is going to react to viewing this type of shape. Another, more controlled, way of creating shapes is by using letters or numbers. Depending on their size, an artist can use the curves and straight lines of a familiar letterform to create new shapes. The artist can even blow up letters or numbers so that they are unrecognizable as their familiar shape. Even blown up to an abnormal size, the familiarity of letters and numbers can create certain moods, capture an audience's attention, or create certain emotions in an audience. Shapes are not just geometric. They can be found in other places such as a photograph of a tree is triangular in shape or the rectangle formed by a group of people standing close together, the rectangle formed by a group of people standing close together, hence, a shape does not have to be just a shape but other objects can be interpreted as seeming to look like a certain shape.

\subsection{Space}

Unlike the other visual aesthetic strategies, space is not something that can be specifically identified. However, in two-dimensional works, an illusion of three-dimensionality can be created and "the spatial depth viewers are led to associate with the images may be as important as the images themselves” (Zelanski \& Fisher, 1996: p. 122).

Artists have several different ways of creating space that viewers will interpret as depth. In "Western culture, if parallel lines seem to converge at a distant vanishing point (an illusion called linear perspective), most viewers will interpret what they see as a composition with great depth" (Zelanski \& Fisher, 1996: p. 23). Artists can then further this sense of depth by contrasting large foreground images with smaller background images because things seen a distance appear to be smaller. Due to atmospheric haze in viewing things at a long distance, the edges of things and contrasts between colors are seen more clearly in close areas. Atmospheric haze is created by giving stronger values and sharp edges to nearby objects and soft edges and softer values to far away objects.

The artist can lead the viewer to interpret the visual work by using a flat sense of space versus a constructed sense of depth. The more flat sense of space will have the audience feel less connected with the subject matter, while the constructed sense of depth will have the audience feeling as if they are within the visual's message.

\subsection{Texture}

Texture is the next strategy that brings the viewer closer to the message in the piece of rhetoric. How we feel 
something when we touch it in reality can be translated into the visual through a variety of techniques. The visual look of what something would feel like if touched may or may not actually correspond with what it really feels like. "We might interpret a skillful pencil drawing of bark as rough texture, even though we know we would feel only the smoothness of the paper and the shallow indentions of the pencil marks if we ran a hand over the drawing" (Zelanski \& Fisher, 1996: p. 151).

One of the easiest ways to create texture is to use actual textures or things that have a noticeable texture. This can be done through collage or by adding materials such as sand to paint to create actual texture on a canvas. "Many artists physically build up paint or other somewhat liquid media to produce rough textures. Oils or acrylics can be applied unthinned so that strokes of the brush or palette knife leave a textured impression. [This] effect, called impasto, can be seen in [Van Gogh's] Starry Night" (Zelanski \& Fisher, 1996: p. 159). Artists also have ways of simulating texture through traditional uses of paint, ink, or graphite. They can use these media to create light and dark shades to suggest textual effects seen in nature.

Texture can also be created that isn't representational or reflective of things in real life. Similar shapes, letters, or lines that are used repeatedly, create a visual illusion of texture. It becomes difficult for us to understand familiar shapes when they are repeated, especially when the shapes are small in size. Type can be used to create texture in a similar manner by repeating letters or words resulting in a texture that can be more important to the design than the legibility of the actual letters or words used. Textures are often used to communicate complexity. If the artist is wanting to relay a complex message, it will appear with layers of visual texture in order to engage the audience within the message.

\subsection{Value}

Value can be defined as the difference in light and dark that appears on any given surface. "Since we see our world in colors, it is initially difficult to extract this quality from all the other qualities of color. But value is of such compositional and conceptual importance that the artist learns to look at things as does a photographer who works in black and white, manipulating areas of darks, lights, and midtones" (Zelanski \& Fisher, 1996: p. 189). Stark emotions can be created in black and white, so the power of using value should not be underestimated by adding color. This can be demonstrated simply by thinking of the nostalgia that one feels when looking at a black and white photograph.

The variations in value ranging from black to white can be compared to the volume of music's loudness and softness. "By this analogy, lines and shapes are the notes and chords played, and [value] becomes the timbre of the instruments. Value is thus a critical element in a fully orchestrated design. A symphony in which all notes were played at the same volume would soon lose the audience's interest” (Zelanski \& Fisher, 1996: p. 189). This comparison shows the necessity of examining value in visual rhetoric.

Light and dark areas of value can be used to direct the audience's eye to a focal point, but neither can automatically draw the attention of the viewer. The emotional responses that can be evoked by values are distinct, but there are no completely set rules on how an audience will be affected or react to a particular value. Strongly contrasting values have a tendency to provoke a more immediate emotional response. A "dark background with light images can evoke a sense of fear, like dreadful dreams in darkness. [Predominantly] light values often evoke a light emotional response, a feeling of happiness. [A] range of mid-values that cannot be mistaken for blacks or whites can convey a sense of hopelessness or depression. [Even] in abstractions in which there is no recognizable image to influence our emotional response, values can affect us strongly" (Zelanski \& Fisher, 1996: pp. 206-210).

\subsection{Color}

The variety of effects and emotional responses created by using value can be even more dynamic with the addition of color. Color is the spice added to the dish of a good visual. Color is a powerful and rich tool, but is also extremely complex. We have a tendency to think of a color in regards to an object, such as "grass green" or "sky blue", but what we see as color is not the object itself instead it is the light reflected off of those objects. There are thousands of variations of colors on the spectrum of refracted light and theorists have come up with many different ways of developing a similar theory of pure colors. "In the seventeenth century, Isaac Newton noticed that the red-purple at one end of the visible spectrum [of light] looked very similar to the purple at the other end. He therefore drew the two ends of the spectrum together, producing the first color wheel" (Zelanski \& Fisher, 
1996: p. 227).

Artists do run into audience's prejudices regarding color as color theories have devised ways to make us feel more comfortable with certain colors and color combinations. In our everyday speech, we use the names of colors to create and reinforce ideas and emotions associated with color. For example, the color yellow is associated with cowardice and green is associated with envy. Yet another road block that faces an artist's use of color is the commonly thought idea that "warm" colors, such as reds and oranges seem to advance in space, while "cool" colors, such as greens and blues seem to recede. "In actuality, any color can be brought forward or pushed back in space by the visual clues to spatial organization given with it" (Zelanski \& Fisher, 1996: p. 238).

Only some of the possibilities of color as a strategy are explored in any of the established color theories. Even with a very limited palette of colors for which to work with, a great number of responses can be coaxed from the audience. "Depending on how you put them together, you can get much more from each color than a flat, unvarying reaction. To equate the possibilities once more with music, imagine the excitement of hearing a basso profundo sing in his lowest range and then hit a very high note. It can happen in music-and it can happen in color" (Zelanski \& Fisher, 1996: p. 250). Color is by far the most powerful and least understood of the strategies.

\subsection{Repetition}

One of the simplest ways of unifying the elements of the visual aesthetic is through repetition, both for the artist to create and for the audience to understand. "Repetition of identical or similar lines, shapes, forms, textures, values, or colors creates a predicable pattern: a coherent visual structure” (Zelanski \& Fisher, 1996: p. 36). We generally respond favorably to designs that are representative of the repetitive patterns that we see in nature. A rose is simply the repetition of the same petal shape. When we see similar elements, they draw our eyes on a path from one to the next, connecting them visually.

Repetition can be as simple as repeating a simple geometric shape or as complex as the understructure of the entire work. Repeated shapes, such as triangles, can provide a solid structure for the work. Artists also develop techniques of repeating strokes, textures, or colors to pull an entire design together. This is demonstrated in Van Gogh's works repetition of stroke length and layering of colors. Repetition in a work creates reliable patterns that the audience is both familiar and comfortable with. This strategy is just one piece of the puzzle in successfully relaying a message from the artist to the audience.

\subsection{Variety}

Inversely, another way of creating unity in a piece of visual rhetoric is to vary the elements used, either as just slight variations of a central theme or with strongly contrasting elements as precise repetition has the potential of boring the viewer. Even though audiences find repetition comfortable, they also enjoy the spice and surprise of variety. "Instead of using slight variations on a unifying theme, artists sometimes present polar opposites-a very rough-textured area contrasted with a very smooth-textured area, a very dark area contrasted with a very light area, a convex shape fitted into a concave shape. Such contrasts challenge the viewer to compare the elements and actively synthesize the contrasting parts by recognizing the relationships between them: They are opposites that seem to complete each other, like the unity of the yin and yang in Chinese philosophy" (Zelanski \& Fisher, 1996: p. 41).

Audiences attempt to make connections between elements as we are uncomfortable with chaotic stimuli. Relationships are thus created out of elements that seem to be polar opposites. "Dark and light are extremes of value; rough and smooth are extremes of texture. A work that contrasts dark and lights may be unified by its references to value; a work that contrasts rough and smooth areas may be unified by its persistent references to texture" (Zelanski \& Fisher, 1996: p. 41). The artist must be able to predict how an audience will connect the variations in a piece and then use that to direct their message and the rhetorical critic must understand the variations used by the artist.

\subsection{Rhythm}

The unifying principle of repetition and variation in any work has the tendency to create a visual rhythm for the viewer to follow while moving through the piece. Elements "tend to be picked up as pulsations, intervals between them as pauses. When the beat is one viewers can sense, it too helps them experience a design as an or- 
ganized, unified whole” (Zelanski \& Fisher, 1996: p. 41).

The artist can utilize this rhythm to direct the viewer to elements and paths through the work in order to further the message for the audience. Again referring back to the music analogy, "visually, a picket fence has the same kind of staccato rhythm as a piece of music in which all notes are of the same duration, equally spaced and equally accented. A work in which lines are more continuous and curving suggests a more flowing rhythm, with gradual crescendos and decrescendos" (Zelanski \& Fisher, 1996: p. 41). Using this analogy, one can almost hear how the audience's emotions can be controlled and advanced through the creation of a rhythmic pattern. With sharp, staccato, angular rhythms, the audience is made to feel on edge and uncomfortable. Whereas, with smooth, regular, undulating rhythms, the audience will feel soothed.

\subsection{Balance}

Another simple strategy that the artist helps the viewer unify elements is through creating a visual balance. Artists "distribute the apparent weight of the elements so that the work does not appear about to topple from being heavier on one side" (Zelanski \& Fisher, 1996: p. 44). The most obvious way to create balance is through symmetry. This type of balance is familiar to the audience as most of what we see around us is appears to be balanced in this symmetrical manner. The left and the right side of things all around us are similar and we find this symmetry comforting. Asymmetrical balance is created when things of differing visual weight are expertly placed to create an appearance of balance. This can be done in a variety of ways, but in "general, the farther a figure is from the center, the more visual weight it suggests. In addition to position and size, visual weight is affected by other factors such as color, value, and degree of detail. In general, areas that are light in value, brightly colored, or highly detailed draw the viewer's attention more than areas that are dark, dull, or less complex; they therefore carry more visual intensity for their size" (Zelanski \& Fisher, 1996: p. 45). In addition to horizontal balance, vertical balance is equally important. In this sense, an absolute symmetry can appear wrong or unbalanced to the viewer. "If a figure is placed precisely in the middle of a two-dimensional surface, [it] may seem too low. Often viewers prefer to see beneath a figure a slightly larger area than above it, giving the impression that it is being adequately supported" (Zelanski \& Fisher, 1996: p. 46).

Balance is an important part of visual rhetoric, but sometimes an artist will intentionally unbalance a piece to create a certain effect or emotion in the audience. It is "nevertheless important to recognize that people do respond to visual weighting in a design, for this is another [strategy the artist] can use to organize the whole in any way [they] intend" (Zelanski \& Fisher, 1996: p. 47).

\subsection{Emphasis}

Emphasis is created through the use of a single focal point or the area that the audience's eye is most compellingly drawn. If there is no focal point drawing the audience's attention, it may feel too chaotic and make it difficult for the audience to comprehend what is going on. There are many ways that a focal point to draw attention can be created. Placing "a figure near the center of a two-dimensional work or slightly above center often helps draw attention to it. Any kind of contrast creates emphasis. In a field of straight lines, a circle will draw attention to itself. In a design that consists of largely dark areas, a light area will stand out; in a group of light colored areas, a dark figure will be emphasized. A large figure may draw more attention than smaller figures" (Zelanski \& Fisher, 1996: p. 48). Scale, or the size relationship of an object to its surroundings, can also be used to draw attention. Larger objects will attract attention more readily in a busy background, while smaller objects will command more attention in a sparse background.

\subsection{Economy}

Economy is the final strategy because even with all of the previous elements, economy is "using only what is needed to create the intended effect, eliminating any elements that might distract attention from the essence of an idea" (Zelanski \& Fisher, 1996: p. 51). Economy can be highly useful in that the audience could potentially spend more time viewing the work than if all the information is presented to them. Abstracted art is one example of how economy can be used in isolating certain elements and focusing more attention on line, shape, value, or color. Economy is the reduction of detail to its simplest expression. For example, a bird in flight can be depicted as a simple, flattened " $v$ " shape. 
The strength of using economy in design is that the artist can create something "using a minimum of visual cues to provoke a complex response is that it involves viewers in the creative experience" (Zelanski \& Fisher, 1996: p. 53). The less the artist shows, the more the audience has to fill in the enthymeme in the work, but we must remember that sometimes less is more.

\section{Conclusion}

The strategies themselves work to give the critic the proper building blocks of understanding of how images are created and how the artist conveys their message to the audience. The addition of the visual rhetorical strategies to the study of rhetoric works to combine the research of aesthetic study, traditional rhetorical studies, and design philosophy. Going into the aesthetic only loses focus on audience and context. Focusing on the rhetorical elements alone loses the visual quality of an artifact. Forging with just a design philosophy loses the elements of what persuasive nature the visual can hold. The combination of these areas can then give rhetorical critics the tools necessary to fully understand and evaluate the images in the world around us. Rhetoric alone cannot understand the visual message and design theory cannot understand the rhetorical implications of the visual message, but the delicate combination of both rhetorical purposes and design principles will give the rhetorical critic the tools necessary to evaluate our ever evolving visual world.

\section{References}

Bernhardt, S. A. (1996). Visual Rhetoric. In T. Enos (Ed.), Encyclopedia of Rhetoric and Composition: Communication from Ancient Times to the Information Age (pp. 746-748). New York: Garland.

Brown, J. C. (1983). Excellence and the Problem of Visual Literacy. Design for Art in Education, 85, 11-13. http://dx.doi.org/10.1080/07320973.1983.9937342

Brummett, B. (1991). Rhetorical Dimensions of Popular Culture. Tuscaloosa: University of Alabama Press.

Fenner, D. E. W. (2003). Aesthetic Experience and Aesthetic Analysis. Journal of Aesthetic Education, 37, 40-53. http://dx.doi.org/10.2307/3527420

Finnegan, C. A. (2003). Picturing Poverty: Print Culture and FSA Photographs. Washington D.C.: Smithsonian Institution Press.

Foss, S. R. (1986). Ambiguity as Persuasion: The Vietnam Veterans Memorial. Communication Quarterly, 34, 326-340. http://dx.doi.org/10.1080/01463378609369643

Foss, S. R. (1994). A Rhetorical Schema for the Evaluation of Visual Imagery. Communication Studies, 45, $213-224$. http://dx.doi.org/10.1080/10510979409368425

Foss, S. K., \& Gill, A. (1987). Michel Foucault's Theory of Rhetoric as Epistemic. Western Journal of Speech Communication, 51, 384-401. http://dx.doi.org/10.1080/10570318709374280

Fuller, P. (1984). Taste-You Can’t Opt out. Design, 423, 38-43.

Haines, H. W. (1986). “What Kind of War?”: An Analysis of the Vietnam Veterans Memorial. Critical Studies in Mass Communication, 3, 1-20. http://dx.doi.org/10.1080/15295038609366626

Jamison, K. H. (1992). Dirty Politics: Deception, Distraction, and Democracy. New York: Oxford University Press.

Kanengieter, M. R. (1991). Message Formation from Architecture: A Rhetorical Analysis. (Doctoral Dissertation, University of Oregon, 1990). Dissertation Abstracts International, 51, 3561A.

Kostelnick, C. (2004). Melting-Pot Ideology, Modernist Aesthetics, and the Emergence of Graphical Conventions: The Statistical Atlases of the United States, 1874-1925. In C. A. Hill, \& M. Helmers (Eds.), Defining Visual Rhetorics (pp. 215242). Mahwah, NJ: Lawrence Erlbaum Associates.

Kostelnick, C., \& Hassett, M. (2003). Shaping Information: The Rhetoric of Visual Conventions. Carbondale, IL: Southern Illinois UP.

Kress, G, \& Van Leeuwen, T. (1996). Reading Images: The Grammar of Visual Design. New York: Routledge.

Langer, S. (1988). Mind: An Essay in Human Feeling (Abridged by G. Van Den Heuvel). London: The Johns Hopkins University Press.

Lauer, D. A., \& Pentak, S. (2000). Design Basics (5th ed.). Fort Worth, TX: Harcourt Brace \& Company.

Lawson, B. (1980). How Designers Think. Westfield, NJ: Eastview Editions, Inc.

Lucaiates, J. L., \& Hariman, R. (2001). Visual Rhetoric, Photojournalism, and Democratic Public Culture. Rhetoric Review, 20, 37-42. 
Margolin, V. (Ed.) (1989). Design Discourse. Chicago, IL: University of Chicago Press.

Moore, P., \& Fitz, C. (1993). Using Gestalt Theory to Teach Document Design and Graphics. Technical Communication Quarterly, 2, 389-410. http://dx.doi.org/10.1080/10572259309364549

Postman, N. (1985). Amusing Ourselves to Death: Public Discourse in the Age of Show Business. New York: Sifton/Penguin.

Rosenfield, L. W. (1989). Central Park and the Celebration of Civic Virtue. In T. W. Benson (Ed.), American Rhetoric: Context and Criticism (pp. 221-266). Carbondale, IL: Southern Illinois University Press.

Twigg, R. (1992). Aestheticizing the Home: Textual Strategies of Taste, Self-Identity, and Bourgeois Hegemony in America’s “Gilded Age”. Text and Performance Quarterly, 12, 1-20. http://dx.doi.org/10.1080/10462939209359630

Zarefsky, D. (1992). Spectator Politics and the Reversal of Public Argument. Communication Monographs, 59, 411-414. http://dx.doi.org/10.1080/03637759209376283

Zelanski, P., \& Fisher, M. P. (1987). Shaping Space. New York: Holt, Rinehart, and Winston.

Zelanski, P., \& Fisher, M. P. (1990). Color. Englewood Cliffs, NJ: Prentice-Hall, Inc.

Zelanski, P., \& Fisher, M. P. (1996). Design Principles and Problems. New York: Harcourt Brace College Publishers. 